0 , IO, and the group of 26 to 27.5 magnetons; and then there is the iron which is nearly as diamagnetic as Pascal (Revue générale des Sciences, July I5, I923) has found bismuth to be in its compounds.

LARS A. WELO.

Rockefeller Institute for Medical Research, New York, July 23.

Mr. Welo's interesting letter directs attention to the fact that the iron atoms in various complex cyanides possess quite different magnetic properties from the iron atoms in simple ferrous or ferric salts. The existence of iron atoms which are either paramagnetic, but have a moment considerably smaller than that found in the simple salts, or even diamagnetic (zero magnetic moment) finds a parallel in the observation of Pascal that oxygen may function in organic compounds as a paramagnetic element with the "usual" moment, as paramagnetic with a lesser moment and also as diamagnetic.

The susceptibilities of a large number of complex co-ordination compounds of cobalt and nickel are known. It would be of considerable interest to carry out similar calculations to Mr. Welo's with these data also. Cobalt and nickel atoms with zero magnetic moment may be expected, but the interesting point would be the determination whether these elements exist in modifications analogous to $\mathrm{Mr}$. Welo's iron with ro magnetons.

It may be of interest to mention the non-magnetic films of nickel which have recently been described. Their existence has been ascribed elsewhere to a rearrangement in the electron configuration similar to that suggested by me in the letter referred to by Mr. Welo. By this rearrangement the electron configuration of the nickel atom becomes completely symmetrical and hence the atom possesses no magnetic moment.

A further study of data such as are given by Mr. Welo and those derived from the simple salts of the magnetic elements is likely to be of considerable interest in giving some evidence of the deformations or rearrangements of the electron orbits of an atom in a solid compound. The magnetic properties of the free undeformed atom may be inferred from spectroscopic data.

Two further points may be mentioned in connexion with Mr. Welo's letter. First, his method of calculation of the magneton numbers implicitly assumes that the various substances obey Curie's law $K T=C$. In general, however, paramagnetic substances obey the more general law, $K(T+\Delta)=C$, so that a single measurement at one temperature is not sufficient for the determination of the Curie constant C, from which the magneton numbers are calculated according to Weiss. Mr. Welo's numbers may, therefore, need a correction on this account. Further, it is scarcely permissible to calculate the Bohr magneton numbers merely by dividing the corresponding Weiss numbers by 5 since, though the Bohr magneton is essentially a quantum unit, no account is then taken of the spatial quantisation factor.

Secondly, it seems that there is a slight misunderstanding of the purpose of my letter already referred to. It is perfectly reasonable to expect that iron atoms in quite different modes of combination should possess different magnetic properties, but in the letter attention was directed to the fact that iron atoms in ferrous compounds may exist in different magnetic states in one and the same compound. Thus the four values of the magneton number there mentioned were obtained from observations on ferrous ammonium sulphate.

L. C. JACKSON.
The Band Spectra associated with Carbon.

HAving read with great interest the two communications by Prof. Raymond T. Birge, published in NATURE of August I, p. I70, and August 8, p. 207, I should like to direct attention to the fact that I gave in I924 the description and wave-lengths of the band spectrum described a year later by Dr. R. C. Johnson as "A New Band System " in Proc. Roy. Soc. A, Io8, 349, June I925, and called " the new Johnson group" by Prof. Birge (NATURE, August 8, p. 207).

My first publication concerning these new bands appeared in Comptes rendus, I78, May 5, I924, p. I 525, under the heading "Sur les spectres de la décharge thermionique dans l'oxyde de carbone. Nouveau spectre de bandes" (italics mine here). It was also published with a photographic reproduction of the bands in L'A stronomie, 38, Nov. I924, 444, Fig. 236.

The thermoelectronic bulb filled with pure carbon monoxide at a pressure of about half a millimetre gives with intensity only the three double-double headed bands at $\lambda 4236,3978,3730$, while the helium mixture of Dr. Johnson gives three additional bands. So far as I can judge with such a limited number of bands, it appears that the distribution of the in tensities seems a high-temperature distribution with thermoelectronic bombardment, while it would be a low-temperature distribution in a helium mixture. Prof. Birge has rightly made the same remark for the comet-tail bands (NATURE, August I, p. I7I).

Since the publication of my above-mentioned papers, I have obtained the three bands with a great dispersion, and I will give later the analysis of their fine structure.

Observatoire de Meudon, August r 2.

\section{The Transport of Organic Foodstuffs in Plants.}

FROM the ringing experiments of early times, it has reasonably been inferred that foodstuffs are translocated downwards by the phloem. But to complete the evidence it would be necessary to prove directly that the tissues external to the wood can translocate foodstuffs by themselves. Adequate experimental evidence for this has never been produced, and recently it has been maintained that downwards as well as upwards the foodstuffs must be translocated by the wood. (Dixon, H. H., and Ball, N. G., NATURE, February 23, I922, vol. I09, p. 236.)

Evidence for translocation by the rind I have, however, now been able to obtain by rooting shoots of Salix fragilis in tap-water, and peeling off from below upwards strips of rind from 4 to $8 \mathrm{~cm}$. long, each carrying a root. The strips remained connected with the shoots above. The roots on these strips grew at rates up to a centimetre a day, and nearly as fast as similar roots on intact parts of the same shoots. They continued to grow indefinitely. The foodstuffs for their growth must have been translocated from the shoot, for roots on short, completely isolated strips of rind grew only for a few millimetres and then stopped, showing that the local supply was insufficient. Since new wood did not begin to be regenerated for from four to eight days, the foodstuffs must have been translocated by the rind alone.

It should be mentioned that before the roots on the strips of rind could be got to grow, it was found necessary either to prevent the water surrounding the roots from touching the inner surfaces of the strips, or to keep this water sterile with one part in 120,000 of thymol.

It is hoped to continue the experiments next spring.

Magdalen College, Oxford.
R. SNOW.

NO. 2914 , VOL. II6] 